\title{
Evaluation of the Patient Profile and Current Pattern of Care with Docetaxel Based Adjuvant Regimen in Operable Breast Cancer
}

\author{
MA Hai ${ }^{1}$, Parveen Shahida Akhter ${ }^{2}$, Quamruzzaman Chowdhury ${ }^{3}$, \\ Parvin Akhter Banu ${ }^{4}$, Mofazzel Hossain ${ }^{5}$, Kumkum Pervin 6
}

\begin{abstract}
Background: Early stage breast cancer can often be cured with surgery alone though there are chances of recurrent disease and decrease in survival. Adjuvant poly-chemotherapy with docetaxel-based regimens can improve both disease-free and overall survival in patients with operable breast cancer. Objective: This study was designed to evaluate the patient profile and current pattern of care with docetaxel based adjuvant regimen in operable breast cancer patients including the treatment outcome from clinical practice. Materials and method: This prospective, observational, non-comparative study planned to enroll newly diagnosed operable breast cancer patients with high risk of recurrence after surgery. Selection of docetaxel-based treatment strategy and dosage of therapy was at the discretion of individual oncologists as per routine clinical practice. Patient data were recorded during inclusion, each cycle of therapy, and follow-up at 1 year (+/- 1 month) after inclusion. Results: Between August 2008 and July 2011 a total of 85 patients with median age of 53 years (23-73 years) were enrolled and 53 (62.4\%) patients were postmenopausal. Ductal carcinoma, the most common cancer type, was found in 73 (85.9\%) patients. Receptor status was positive for estrogen, progesterone or Her2/neu/erbB2 in $65.9 \%, 47.1 \%$ and $5.8 \%$ patients, respectively. Mastectomy either partial or total was performed in $76(89.4 \%)$ patients for the current disease. An average of 8 (range: 2-15) lymph nodes (LN) mostly in axilla were excised in 56 patients and average of 4 (range: 1-11) LN nodes were positive. Sentinel LNs were negative, excised in 4 patients. The mean tumor size was 5.5 $\mathrm{cm}$ and most (82.4\%) patients were diagnosed at Stage II disease including some at Stage I and III (7.1\%, 10.6\%). Docetaxel (Taxotere $\left.{ }^{\circledR}\right)$ was combined with cyclophosphamide alone or in addition with doxorubicin, epirubicin, $5 \mathrm{FU}$ and trastuzumab either in combination or sequential regimen. Taxotere in combination with adriamycin and cyclophosphamide was planned for $63.5 \%$ patients. Trastuzumab as sequential therapy could be afforded by 1 patient only. Data regarding radiotherapy or hormone therapy was not recorded. At the end of four cycles, $83.5 \%$ of patients were found disease free and 5.9\% had loco regional relapse. At 1 year 71 (93.4\%) patients were alive, 63 (88.7\%) were relapse-free and 8 (11.2\%) had loco regional relapse.
\end{abstract}

1. Director, Bangladesh Cancer Hospital and Welfare Home, Dhaka, Bangladesh.

2. Consultant, Shanti Oncology Center and Ex-Comsultant of National Institute of Cancer Research \& Hospital, Dhaka, Bangladesh.

3. Ex-Professor of National Institute of Cancer Research \& Hospital, Consultant of Oncology in Bangladesh Specialized hospital, Dhaka, Bangladesh.

4. Senior Consultant of Oncology, Delta Medical College \& Hospital, Dhaka, Bangladesh.

5. Consultant of Oncology, Lab Aid Specialized Hospital, Dhaka, Bangladesh.

6. Affiliate Pharmacovigilance, Head of Sanofi Bangladesh Limited, Dhaka, Bangladesh.

Correspondence: Dr. Parvin Akhter Banu. e-mail: Pabanu@yahoo.com 
Nausea, vomiting, diarrhea, alopecia, anemia and neutropenia were most commonly reported adverse events classified as Grade 1 or Grade 2. Grade 3 neutropenia was reported in 5 patients and 2 patients reported grade 4 neutropenia. Grade 3 diarrhea was reported in 1 patient. Conclusion: Docetaxel as adjuvant chemotherapy offered one year survival in $93.4 \%$ (71/76) of the patients and $88.7 \%$ (63/71 patients) were disease-free. The safety profile of docetaxel based regimens was expected and manageable.

Keywords: Docetaxel; Adjuvant regimen; Breast cancer.

Delta Med Col J. Jan 2019;7(1):4 - 10

\section{Introduction}

Breast cancer is the most frequently diagnosed cancer and leading cause of cancer death among women, accounting for $25 \%$ of the total cancer cases (1.68 million) and $15 \%$ of cancer deaths $(522,000)$ worldwide. ${ }^{1,2}$ Localized and regionally advanced breast cancer is potentially curable with local and systemic therapy. Adjuvant systematic therapies are currently administered with the goal of eradicating microscopic foci of cancer cells that, if untreated, could grow and recur as metastatic cancer. In the early 20 th century radical mastectomy was the standard surgical procedure for breast cancer treatment. ${ }^{3}$ Over time a series of clinical trials demonstrated that adjuvant systemic chemotherapy, endocrine therapy, and anti-HER2-directed therapy substantially reduced the risk of recurrence and improved overall survival when added to local therapy. ${ }^{4}$ The Early Breast Cancer Trialists' Collaborative Group (EBCTCG) has periodically reported meta-analyses of all clinical trials with available data that have added the benefits of adjuvant systemic therapy in breast cancer treatment. ${ }^{5-9}$

If screening programs and guidelines for the early detection of breast cancer are well adopted, it will improve the chances that breast cancer can be diagnosed at an early stage, treated successfully, and save many thousands of lives each year. Unfortunately, screening of breast cancer is not widely available in Bangladesh or in all Asia-Pacific countries. ${ }^{10}$ There is a lack of local data in Bangladesh concerning clinical practice of newly diagnosed breast cancer patients. This study was designed to evaluate the patient profile and current pattern of care with docetaxel based adjuvant regimen in operable breast cancer patients including the treatment outcome from clinical practice.

\section{Materials and method}

This was a prospective, observational, non-comparative, non-interventional registry study on evaluation of the patient profile and current pattern of care with docetaxel-based adjuvant regimens in operable breast cancer. A total of 85 patients were enrolled between August 2008 and July 2011. Eligible patients were female, more than 18 years of age, who were newly diagnosed with breast cancer, and having a high risk of recurrence after surgery i.e. node positive cases, or node negative cases where the physician assumed association of high risk of recurrence considering tumour size, pathologic grade, receptor status, and age of the patient. Patients who had prior therapy other than surgery for the current disease or had history of hypersensivity to docetaxel or other drugs formulated with polysorbate 80 were not eligible for this study. Patients with a neutrophil count less than 1500 cells $/ \mathrm{mm}^{3}$, serum level of bilirubin above the upper limit of normal (ULN), SGOT and/or SGPT more than $1.5 \mathrm{x}$ ULN concomitant with alkaline phosphatase more than $2.5 \times$ ULN were also excluded.

Upon informed consent, 50 patients were planned to be enrolled consecutively by 10 oncologists in this registry study. As this was a non-interventional study, the selection of 
treatment strategy and dosage of therapy were at the discretion of the individual oncologist as per routine clinical practice. The investigators recorded patient data using a paper copy of the Data Collection Forms (DCF) during inclusion, each cycle of therapy, and follow-up at 1 year (+/1 month) after inclusion. At the inclusion visit, patient demographics, relevant medical history, survey specific malignancy including breast cancer stage, biologic characteristics (receptor status, HER2, pathologic grade and other markers), curative anti-cancer surgery, and chemotherapy plan were recorded to describe the patient profile and pattern of care in the patients. During each cycle the therapy received, concomitant medications, and adverse events (AEs) reported by the patient were recorded. At the end of adjuvant therapy the reason for treatment discontinuation (completed prescribed dosing, disease relapse/recurrence, adverse event, death, survey physician/patient refusal, other) was recorded. During follow-up visits, patient survival and disease relapse or recurrence status were recorded.

The number of patients was determined from the estimated proportion of operable breast cancer patients with high risk of recurrence treated with docetaxel-based adjuvant regimens in Bangladesh. To get the optimum number of patients, and considering non-responder, drop out and design effects, 50 patients were planned to be initially included in this registry. However, as planned enrolment was completed 8 months before the planned end of recruitment, the investigators decided to enroll 4 more patients at each site. All the enrolled patients were considered for evaluating patient profile and treatment pattern. Among the patients who completed 4 cycles of docetaxel-based adjuvant chemotherapy were considered for treatment outcome analysis evaluated by survival and disease status (relapse/recurrence) 1 year after treatment. Serious AEs, if any, were recorded by the physician, including date of onset, maximal intensity, action taken with respect to chemotherapy, corrective therapy given, and outcome. Non-serious AEs were recorded on a checklist by worst toxicity grade on a scale from 1-4 according to National Cancer Institute (NCI) Common Toxicology Criteria Manual Version 3.0. Data were expressed as mean, median, standard deviation, minimum, maximum and percentages using SPSS 17.0

\section{Results}

The median age of the patients was 53 years with the youngest at 23 and oldest at 73 years of age. More than half $(62.4 \%)$ of the patients were postmenopausal and $5(5.9 \%)$ presented with prior history of ductal carcinoma in situ (DCIS). The majority $(81 \%)$ of the patients were from urban areas, $27(31.8 \%)$ were overweight (BMI> $25 \mathrm{~kg} / \mathrm{m} 2)$ and $4(4.7 \%)$ had co-morbid diabetes mellitus or cardiovascular disease. The basic demography of the patients and relevant medical history are presented in Table I and II.

Table I: Basic demography of the patients $(\mathrm{N}=85)$

\begin{tabular}{lcccc}
\hline Variables & Mean \pm SD & Median & Minimum & Maximum \\
\hline Age (years) & $51.48 \pm 8.99$ & 53.0 & 23 & 73 \\
Height $(\mathrm{cm})$ & $157.75 \pm 7.41$ & 158.0 & 145 & 185 \\
Weight $(\mathrm{kg})$ & $58.07 \pm 6.94$ & 58.0 & 43 & 72 \\
BMI $\left(\mathrm{kg} / \mathrm{m}^{2}\right)$ & $23.36 \pm 2.66$ & 23.1 & 18.4 & 28.0 \\
\hline
\end{tabular}

Table II: Relevant medical history of the patients $(\mathrm{N}=85)$

\begin{tabular}{lcc}
\hline Medical history & Central & Percentage (\%) \\
\hline History of proliferative breast disease or hyperplasia & 1 & 1.2 \\
History of other types of cancer & 1 & 1.2 \\
Family history of breast cancer & 1 & 1.2 \\
Family history of ovarian cancer & 1 & 1.2 \\
Associated co-morbidities & & \\
$\quad$ Diabetes & 3 & 3.5 \\
Cardiovascular disease & 1 & 1.2 \\
and/or thromboembolic events & & \\
Menopausal status & & \\
Postmenopausal & 53 & 62.4 \\
Premenopausal & 26 & 30.6 \\
Perimenopausal & 6 & 7.1 \\
\end{tabular}

The initial breast cancer was detected by clinical breast examination $(62.4 \%)$ by the physician and mammogram (58.8\%) whereas breast selfexamination was also the reason for initial detection in almost half $(50.6 \%)$ the patients. 
Table III: Method of cancer diagnosis, histology and receptor status in patients $(\mathrm{N}=\mathbf{8 5})$

\begin{tabular}{lc}
\hline Variables & Frequency ( Percentage) \\
\hline Method of initial detection & $43(50.6)$ \\
Breast self-examination & $53(62.4)$ \\
Clinical breast examination & $50(58.8)$ \\
Mammogram & \\
Methods of confirmation of diagnosis & $72(84.7)$ \\
Fine needle aspiration cytology & $17(20.0)$ \\
Excisional biopsy & $1(1.2)$ \\
Incisional biopsy & $3(3.5)$ \\
Core biopsy & \\
Histopathological appearance & $19(22.4)$ \\
Well differentiated & $40(47.1)$ \\
Moderately differentiated & $17(20)$ \\
Poorly differentiated & $9(10.6)$ \\
Undifferentiated & \\
Histological type of tumor & $73(85.9)$ \\
Ductal carcinoma & $6(7.1)$ \\
Lobular carcinoma & $1(1.2)$ \\
Mixed carcinoma & $5(5.9)$ \\
Unknown & $56(65.8)$ \\
Hormone receptor and & biological \\
marker status & \\
ER & \\
Positive & $16(18.8)$ \\
Negative & \\
Unknown & \\
Negitive & \\
Negative & \\
\hline
\end{tabular}

Fine needle aspiration (FNA) was performed in a majority of the patients $(84.7 \%)$ to confirm initial diagnosis. Excisional biopsy (20.0\%), core biopsy (3.5\%) and incisional biopsy (1.2\%) were also done. Ductal carcinoma was the most common histopathological cancer type found in 73 (85.9\%) patients and $22.4 \%$ of the tumors were well differentiated, $47.1 \%$ moderately differentiated and $20 \%$ were poorly differentiated. The degree of tumor proliferation was measured in 68 patients and $74.1 \%$ were categorized as intermediate and $5.9 \%$ as low. Estrogen and progesterone receptor was positive in $56(65.9 \%)$ and 40 (47.1\%) patients, respectively, and positive Her2/neu/erbB2 was reported in $5(5.8 \%)$ patients. The method of cancer diagnosis, histology and receptor status are shown in Table III. The risk of recurrence or relapse within the next 5 years as assessed on physicians' subjective estimation was high in $9.4 \%$ (8) patients, intermediate in $54.1 \%$ (46), low $24.7 \%$ (21) and low or minimal in $9.4 \%$ (8) patients.

The majority $(82.4 \%)$ of the patients were diagnosed at Stage II disease, 6 patients $(7.1 \%)$ were in Stage I and $9(10.6 \%)$ were in Stage III. Tumor staging in the patients are presented in Table IV.

\section{Table IV: Tumor staging in patients $(\mathbf{N}=\mathbf{8 5})$}

\begin{tabular}{lcc}
\hline Variables & Frequency & Percentage (\%) \\
\hline Stage of tumor & 1 & 1.2 \\
T0 & 16 & 18.8 \\
T1 & 41 & 48.2 \\
T2 & 27 & 31.8 \\
T3 & & \\
Nodal Status & 28 & 32.9 \\
N0 & 51 & 60.0 \\
N1 & 6 & 7.1 \\
N2 & & \\
Overall stage of disease & 6 & 7.1 \\
Stage I & 70 & 82.4 \\
Stage II & 9 & 10.6 \\
Stage III & & \\
\hline
\end{tabular}

Prior to enrolment in the study, 28 patients had breast surgery; partial mastectomy in 24 and total mastectomy in 4 patients. The mean tumour size was $5.5 \mathrm{~cm}$ and the surgical margin was positive in 10 patients $(41.7 \%)$. Eighteen $(21.2 \%)$ of the patients had axillary lymph node excision with an average of 9 (range: 1-15) nodes excised. Among those, $13(76.5 \%)$ patients had positive nodes with an average of 2 (range: 1-8) lymph nodes positive. Sentinel lymph node excision was done in 1 patient with a negative finding. Microscopically, vascular invasion was found in 12 cases and lymphatic vessel invasion in 10 cases. Six (25\%) of the patients received partial breast radiation therapy before surgery.

Surgery for the current breast cancer was performed in $76(89.4 \%)$ patients after enrolment; 
$16(21.1 \%)$ patients underwent partial mastectomy (unilateral-15, bilateral-1) and 60 patients (78.9\%) underwent total mastectomy with or without reconstructive surgery. Axillary lymph nodes were excised in $56(65.9 \%)$ patients and $1(1.2 \%)$ patient underwent internal mammary lymph nodes excision with an average of 8 (range: 2-15) lymph nodes excised and average of 4 (range: 1-11) lymph nodes positive. Sentinel lymph nodes were excised in $4(4.7 \%)$ patients and the nodes were negative. The mean tumour size was $5.5 \mathrm{~cm}$ and 26 patients $(34.2 \%)$ had positive final surgical margin. Microscopically, vascular invasion was found in 43 cases $(56.6 \%)$ and lymphatic vessel invasion was found in 42 cases $(55.3 \%)$.

For treating the patients, docetaxel-based combination therapy was prescribed by the physicians to 71 patients $(83 \%)$ and docetaxel sequential therapy to 14 patients $(16.5 \%)$. Docetaxelin combination with adriamycin and cyclophosphamide (TAC) was prescribed for majority $(63.5 \%)$ of the patients. The prescribed regimen of chemotherapy is shown in Table V. Docetaxel was combined with cyclophosphamide in all the patients, with doxorubicin in $68.2 \%$ of patients, and with epirubicin in $23.5 \%$ of patients. Docetaxel was also combined with 5 FU (11.8\%) and trastuzumab $(1.2 \%)$.

\section{Table V: Chemotherapy in patients}

\begin{tabular}{lcccc}
\hline Chemotherapy & $\begin{array}{c}\text { Stage I } \\
(\mathbf{n = 6})\end{array}$ & $\begin{array}{c}\text { Stage II } \\
(\mathbf{n}=\mathbf{7 0})\end{array}$ & $\begin{array}{c}\text { Stage III } \\
(\mathbf{n}=\mathbf{9})\end{array}$ & $\begin{array}{c}\text { Total } \\
(\mathbf{n}=\mathbf{8 5})\end{array}$ \\
\hline Docetaxel combination regimen & & & & \\
TAC & $2(33.3)$ & & $7(77.8)$ & $54(63.5)$ \\
TEC & - & $9(12.9)$ & $1(11.1)$ & $10(11.8)$ \\
TC & $2(33.3)$ & $5(7.1)$ & - & $7(8.2)$ \\
Docetaxel sequential regimen & & & & \\
FEC followed by Docetaxel & $2(33.3)$ & $8(11.4)$ & - & $10(11.8)$ \\
AC followed by Docetaxel & - & $2(2.9)$ & $1(11.1)$ & $3(3.5)$ \\
AC followed by Docetaxel and Trastuzuma & - & $1(1.4)$ & - & $1(1.2)$ \\
\hline
\end{tabular}

[T:Taxotere, A:Adriamycin (doxorubicin), C: Cyclophosphamide, E: Epirubicin, F: 5-flurouracil]

All the enrolled patients received docetaxel-based chemotherapy either in combination or sequential regimen. Among those, 76 (89.4\%) received 4 cycles of docetaxel based adjuvant chemotherapy and were considered for outcome analysis. Nine patients $(10 \%)$ were not able to continue the chemotherapy after second cycle due to financial limitation. At the end of 4 cycles of adjuvant chemotherapy $71(93.4 \%)$ patients were found disease-free and $5(6.5 \%)$ patients had loco regional recurrence or relapse. On evaluation during 1 year follow-up 71 (93.4\%) patients were surviving, $63(88.7 \%)$ were relapsefree and 8 (11.2\%) patients had experienced loco regional disease relapse. Data for 5 patients $(6.5 \%)$ were not available for the 1 year evaluation and considered as lost to follow-up.

Adverse events were reported in 42 (49.4\%) patients, among which, nausea $(73.8 \%)$, vomiting $(61.9 \%)$, diarrhea $(38.0 \%)$ and alopecia $(33.3 \%)$ were most common. Anemia (61.9\%) and neutropenia $(59.5 \%)$ were most common hematological abnormalities reported (Table VI). No AEs led to patient withdrawal from the study. According to physicians' assessment $25 \%$ of the reported adverse events were related to the chemotherapy. No serious AEs were reported by the physicians. Most of the reported AEs were classified as Grade 1 or Grade 2. Grade 3 neutropenia was reported in 5 patients and 2 patients reported grade 4 neutropenia. Grade 3 diarrhea was also reported in 1 patient.

Table VI: Summary of adverse events related with chemotherapy

\begin{tabular}{lccc}
\hline \multirow{2}{*}{ Adverse events } & \multicolumn{3}{c}{ Grades* } \\
\cline { 2 - 4 } & All & $\mathbf{3}$ & $\mathbf{4}$ \\
\hline Febrile Neutropenia & $8(19.0)$ & - & - \\
Neutropenia & $25(59.5)$ & $5(11.9)$ & $2(4.8)$ \\
Thrombocytopenia & $5(11.9)$ & - & - \\
Anaemia & $26(61.9)$ & - & - \\
Neutropenic infection & $5(11.9)$ & - & - \\
Nausea & $31(73.8)$ & - & - \\
Vomiting & $26(61.9)$ & - & - \\
Stomatitis & $3(7.1)$ & - & - \\
Diarrhea & $16(38.0)$ & $1(2.4)$ & - \\
Asthenia & $5(11.9)$ & - & - \\
Alopecia & $14(33.3)$ & - & - \\
Amenorrhea & $2(4.7)$ & - & - \\
Edema & $7(16.7)$ & - & - \\
Myalgia & $9(21.4)$ & - & - \\
Neuropathy & $5(11.9)$ & - & - \\
\hline
\end{tabular}

*National Cancer Institute Common Terminology Criteria of Adverse Events Version 3.0 


\section{Discussion}

In early breast cancer adjuvant chemotherapy has been shown to be beneficial in improving survival and patient's quality of life. Selection of appropriate agents for adjuvant therapy depends on patient characteristics as well as prognostic and predictive factors. ${ }^{11}$ Nodal status, tumour size, histological type, grade, hormone-receptor status and age are some of the key factors affecting the treatment strategy and clinical outcomes for early breast cancer patients. ${ }^{12}$ Various studies reported important epidemiological and treatment approaches differences in early breast cancer in Asian populations compared with their western counterparts. ${ }^{13}$ Anthracyclines were the gold standard adjuvant therapy but in newer approaches a docetaxel-based regimen and sequential therapy shows greater response. Hence, it warrants a systematic in-depth observational investigation of the safety and efficacy of current chemotherapeutic agents such as anthracyclines and taxanes in the real-world. In this context, this prospective, observational, non-comparative, non-interventional registry was designed to evaluate the patient profile and current pattern of care with a docetaxel-based adjuvant regimen in operable breast cancer in Bangladesh. This registry study was carried out to evaluate patient profile and treatment pattern with docetaxel based adjuvant regimen in patients with operable breast cancer. Initially, 50 patients were planned to be enrolled in a period of 2 years but the investigators decided to enroll 35 more patients as the targeted enrolment was completed 8 months before the planned timeline. The median age of the patients was 53 years with the youngest at 23 and eldest at 73 years of age. Only 5 (5.9\%) patients presented with prior history of ductal carcinoma in situ (DCIS). But unlike the other countries of Asia pacific, clinical breast examination was the common method for detection of initial breast cancer where breast self-examination is higher in other countries $(80 \%),{ }^{13}$ indicating overall social awareness on breast cancer is still low in Bangladesh.
To establish cancer diagnosis, fine needle aspiration (FNA) was done in a majority of the patients $(83.5 \%)$. Histologically $22.4 \%$ of the tumors were well differentiated, $47.1 \%$ moderately differentiated and $20 \%$ were poorly differentiated. Ductal carcinoma was the major $(85.9 \%)$ histological type including lobular carcinoma $(7 \%)$ and mixed carcinoma $(1.2 \%)$. If we look into the histological type of cancer we can see around $73(85.9 \%)$ patients having ductal carcinoma and only 6(7.1\%) patients having lobular carcinoma which is quite similar to other Asian countries. ${ }^{13}$ Though an observational study with docetaxel-based therapy needs long term follow up (like 3 years or 5 years) to evaluate actual disease free survival status, overall survival and relapse rate, in this study we have followed 1 year response rate but the result is quite similar with same type of real life study in adjuvant setting on Asia population. On evaluation during 1 year follow-up 71 patients $(83.5 \%)$ were alive with 63 patients $(82 \%)$ without relapse of the disease. Eight patients (10\%) had loco-regional disease relapse. The relapse rate was non-significant in patients with later stage disease and was comparable to an observational cohort study with 1542 patient showing 16.3\% relapse rate after 5 years follow up with the disease-free survival (DFS) rate was $82 \%$ and overall survival (OS) rate was $91 \%$ at the end of 5-year follow-up. ${ }^{13}$

Nausea, vomiting, diarrhea and alopecia were the most commonly reported adverse events and anemia and neutropenia were the most common hematological abnormalities. Most of the reported adverse events were classified as Grade 1 or Grade 2. Grade 3 neutropenia was reported in 5 patients and 2 patients reported grade 4 neutropenia. Grade 3 diarrhea was reported in 1 patient. No serious adverse events were reported by the physicians. No adverse events lead the patients to withdraw from the study. The incidence of AEs or SAEs was found to be comparable with the data available from various phase III clinical trials. ${ }^{13}$ 


\section{Conclusion}

In this registry $93.4 \%$ of the patients received 4 cycles of docetaxel based adjuvant therapy and were alive after one year and $88.7 \%$ of were disease free. The safety profile of docetaxel-based regimens was expected and manageable. Nausea, vomiting, diarrhea, and alopecia were most common adverse events whereas anemia and neutropenia were most common hematological abnormalities. No adverse event was serious or led to patient withdrawal from the study.

\section{Acknowledgement}

The study was supported by Sanofi Bangladesh Limited. The authors were investigators of this study and received investigator fees from Sanofi Bangladesh for conducting the study. There was no other potential conflict of interest.

\section{References}

1. Jemal A, Center MM, DeSantis C, Ward EM. Global Patterns of Cancer Incidence and Mortality Rates and Trends. Cancer Epidemiol Biomarkers Prev. 2010;19:1893-907.

2. Torre LA, Bray F, Siegel RL, Ferlay J, Lortet-Tieulent J, Jemal A. Global Cancer Statistics, 2012. CA Cancer J Clin. 2015;65:87-108.

3. Cotlar AM, Dubose JJ, Rose DM. History of Surgery for Breast Cancer: Radical to the Sublime. Curr Surg. 2003;60:329-37.

4. Anampa J, Makower D, Sparano JA. Progress in Adjuvant Chemotherapy for Breast Cancer: An Overview. BMC Medicine. 2015;13:195.

5. Early Breast Cancer Trialists' Collaborative Group. Effects of Adjuvant Tamoxifen and of Cytotoxic Therapy on Mortality in Early Breast Cancer. An Overview of 61 Randomized Trials among 28,896 Women. N Engl J Med. 1988;319(26):1681-92.
6. Early Breast Cancer Trialists' Collaborative Group. Tamoxifen for Early Breast Cancer. Cochrane Database Syst Rev. 2001;1:CD000486.

7. Early Breast Cancer Trialists' Collaborative Group. Effects of Chemotherapy and Hormonal Therapy for Early Breast Cancer on Recurrence and 15-Year Survival: An Overview of the Randomised Trials. Lancet. 2005;365:1687-717.

8. Davies C, Godwin J, Gray R, Clarke M, Cutter D, Darby S, et al. Early Breast Cancer Trialists' Collaborative Group (EBCTCG). Relevance of Breast Cancer Hormone Receptors and Other Factors to the Efficacy of Adjuvant Tamoxifen: Patient-Level Meta-Analysis of Randomised Trials. Lancet. 2011;378:771-84.

9. Peto R, Davies C, Godwin J, Gray R, Pan HC, Clarke M, et al. Early Breast Cancer Trialists' Collaborative Group (EBCTCG). Comparisons between Different Polychemotherapy Regimens for Early Breast Cancer: Meta-Analyses of Long-Term Outcome among 100,000 Women in 123 Randomised Trials. Lancet. 2012;379:432-44.

10. Wu TY, Guthrie BJ, Bancroft JM. An Integrative Review on Breast Cancer Screening Practice and Correlates among Chinese, Korean, Filipino, and Asian Indian American Women. Health Care Women Int. 2005;26:225-46.

11. globocan.iarc.fr [Internet]. GLOBOCAN 2012. Estimated Cancer Incidence, Mortality and Prevalence Worldwide in 2012 [cited 2014 Nov 3]. Available from: http://globocan.iarc.fr/Pages/fact_ sheets_cancer.aspx.

12. Mincey BA, Palmeri FM, Perez EA. Adjuvant Therapy for Breast Cancer: Recommendations for Management Based on Consensus Review and Recent Clinical Trials. Oncologist. 2002;7:246-50.

13. Kim SB, Sayeed A, Villalon AH, Shen ZZ, Shah MA, Hou MF, et al Docetaxel-Based Adjuvant Therapy for Breast Cancer Patients in Asia-Pacific Region: Results from 5 Years Follow-Up on Asia-Pacific Breast Initiative-I. Asia-Pacific Journal of Clinical Oncology. 2016;12:125-32. 